\title{
Long-term efficacy of subtotal splenectomy due to portal hypertension in cirrhotic patients \\ CrossMark
}

\author{
Haibo $\mathrm{Chu}^{1 \dagger}$, Wei Han ${ }^{1 \dagger}$, Lei Wang ${ }^{2}$, Yongbo Xu' , Fengguo Jian $^{3}$, Weihua Zhang ${ }^{4}$, Tao Wang ${ }^{5}$ and Jianhua Zhao ${ }^{\text {* }}$
}

\begin{abstract}
Background: Portal hypertension (PHT) requires invasive measures to prevent rupture and bleeding of esophagogastric varices; however, the long-term results of subtotal splenectomy plus fixation of the retrosternal omentum majus (SSFROM) have not been reported. Specifically, the advantages and disadvantages of surgery that preserves the spleen and the long-term hematologic effects have not been described.
\end{abstract}

Study design: Our studies relating to SSFROM commenced in February 1999. As of April 2014 we have performed 256 subtotal splenectomies The records of 65 patients with PHT who underwent SSFROM were reviewed retrospectively.

Results: Four patients died within 4 years of surgery, with a 4-year survival rate of $94 \%$; the 11 -year survival rate was $60 \%$. Eleven patients (17\%) had re-bleeding from esophagogastric varices. The white blood cell and platelet counts were higher 6 and 11 years post-operatively compared with pre-operative values $(P<0.01)$. Portal venous diameter, portal venous flow volume, splenic artery flow volume, as well as splenic length, thickness, and average cross-sectional areas were shown to be significantly constricted or decreased $(P<0.01)$. The proportion of serum CD3+ T cells, CD4+ T cells, and CD8+ T cells was increased $(P<0.01)$, while the serum levels of macrophage colony-stimulating factor and granulocyte-macrophage colony-stimulating factor were significantly decreased $(P<0.01)$. There was no significant change in the serum levels of $\lg \mathrm{A}, \lg \mathrm{M}, \lg \mathrm{G}$, and Tuftsin $(P>0.05)$. DSA demonstrated that 15 cases formed collateral circulations between the portal vein and superior vena cava.

Conclusion: SSFROM provide long-term hemostasis for esophagogastric variceal bleeding in PHT and corrected hypersplenism. SSFROM is an effective treatment for patients with PHT in whom long-term survival is expected.

Keywords: Portal hypertension, Splenomegaly, Subtotal splenectomy, Shunt

\section{Background}

The incidence of portal hypertension (PHT) with posthepatitis cirrhosis is higher in China than elsewhere worldwide. PHT with concurrent esophagogastric variceal bleeding is one of the common causes of early death [1]. Vein ligation under endoscopy and injection sclerotherapy are the preferred hemostasis methods, and bleeding control rates are 89.7 and $87.5 \%$, respectively $[2,3]$. A switch to decompressive shunt procedures is mandatory if endoscopic therapy fails to control recurrent variceal hemorrhage [4]. We rely on

\footnotetext{
*Correspondence: jianhuazhaowf@163.com

'Equal contributors

'Center of General Surgery, The 89th Hospital of People's Liberation Army, West Beigong Road 256, Weifang 261021, China

Full list of author information is available at the end of the article
}

bionics to devise a new technique to treat PTH with subtotal splenectomy plus fixation of the retrosternal omentum majus (SSFROM) [5]; however, this technique is controversial. In the present study, the re-bleeding rate, survival rate, hemodynamics, hematologic parameters, splenic function, immunologic parameters, and related indices were analyzed retrospectively to evaluate the longterm efficacy of SSFROM in patients with PHT.

\section{Methods}

Ethical approval of the study protocol (No. 1685) was obtained from the Human Research Ethics Committee of the $89^{\text {th }}$ Hospital of PLA (Weifang, China). All individuals provided written informed consent before enrollment in the study. 


\section{Study population}

Our studies relating to SSFROM commenced in February 1999. As of April 2014 we have performed 256 subtotal splenectomies (preserving the lower pole, normal spleen size of splenic tissue, splenic omentum, and splenocolic vessels for the purpose of blood supply). Sixty-five patients (34 males and 31 females; mean age, 34 years; age range, 25-43 years) with splenomegaly due to PHT were randomly selected; 13 patients underwent emergency surgery and 52 patients underwent elective surgery. Liver biopsies were performed on all patients to confirm cirrhosis. Twenty-three patients had bleeding episodes prior to surgery and underwent endoscopic injection sclerotherapy pre-operatively. Six patients underwent six sessions of endoscopic injection sclerotherapy. Patients were confirmed to have cirrhosis after hepatitis-B infection, HBV-DNA was negative, and were class A or B according to the Child-Pugh classification. Cirrhosis was accompanied by hypersplenism with moderate or severe varicose veins of the lower esophagus, plus a history of digestive tract hemorrhage. All patients were followed up continuously.

\section{Operative method}

A left upper abdominal " $L$ " incision was made in the abdomen to explore the liver and spleen. First, the lienorenal and splenophrenic ligaments were ligated. Then, the spleen was moved through the incision. Second, the gastrosplenic ligament and splenic artery stem were ligated. The left gastroepiploic and splenocolic vessels were preserved (Fig. 1a). Third, along the ischemic separatrix the subtotal spleen was removed; the spleen bed was sutured by serous membranization. Residual spleen, $11 \times 7 \times 4 \mathrm{~cm}$ in size, was preserved. The residual splenic section was sutured using a horizontal mattress cross-suture method (Fig. 1b). Fourth, the splenic capsule was cauterized with an electrotome to create a rough surface. Pericardial devascularization was performed. Fifth, the omentum majus $(10 \times 5 \mathrm{~cm}$ and $10 \times 10 \mathrm{~cm}$ ) was excised and the retrosternal space was separated. A part of the omentum majus was

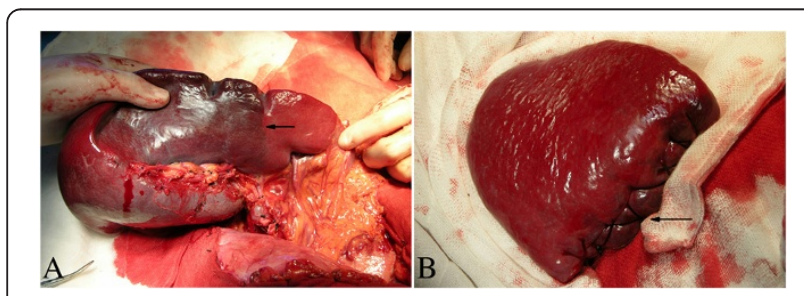

Fig. 1 a The lienorenal and splenophrenic ligaments were ligated. then, the gastrosplenic ligament and splenic artery stem were ligated. The ischemic separatrix occurred obviously in spleen (arrow). b The residual splenic section was sutured using a horizontal mattress cross-suture method (arrow) tamponaded into the retrosternal space and fixed in place. The residual spleen and the other omentum majus were fixed into the retroperitoneum. Drainage catheters were placed in the splenic recess, and the abdominal cavity was closed.

\section{Evaluation}

The cumulative survival and non-bleeding rates were determined by the Kaplan-Meier method. The portal venous diameter, portal venous flow volume, and splenic artery flow volume were detected by colour Doppler sonography. The peripheral white blood cell and platelet counts were measured using a hematology analyzer. An abdominal CT scan measured the splenic size (splenic length, splenic thickness, and splenic square area). Splenic scintigraphy with ${ }^{99 \mathrm{~m}} \mathrm{Tc}$ sulfur colloid evaluated the phagocytic function of the residual spleen by ECT. The serum CD3+ T cells, CD4+ T cells, and CD8+ T cells were enumerated by flow cytometry. Serum macrophage colonystimulating factor (M-CSF) and granulocyte-macrophage colony-stimulating factor (GM-CSF) were measured by ELISA. Serum Tuftsin was measured with a radioimmunoanalyzer. Serum IgA, IgM, and IgG were measured with a fully automatic biochemical immune analyzer. Using digital subtraction angiography (DSA) through an indwelling catheter in vessels of the omentum majus post-operatively, the collateral vascular shunt between the portal vein and superior vena cava was observed.

\section{Statistical analyses}

Data analyses were performed using SPSS (version 17.0; SPSS, Inc., Chicago, IL, USA). All values are the mean \pm standard deviation. Groups were compared using a t-test. A $P<0.05$ was considered significant.

\section{Results}

\section{Survival rate}

The 4- and 11-year survival rates were 94 (61/65) and $60 \%(39 / 65)$, respectively. Four patients died. Two patients survived for 152 and 278 days. The cause of death was complete occlusion of the portal vein as a result of portal thrombosis. Two patients survived for 2 and 4 years. The cause of death was hepatic carcinoma complicating liver failure (Fig. 2a).

\section{Recurrent bleeding rate}

Post-operative recurrent bleeding occurred in 11 patients (17\%), due to esophageal varices in eight patients and from a gastric varix in three patients. All of the patients were cured with conservative therapy. Of the 11 patients who bled post-operatively, seven had a history of pre-operative bleeding and four were complicated with portal thrombosis post-operatively. The 4- and 10 -year non-bleeding rates were both $83 \%(54 / 65$; 


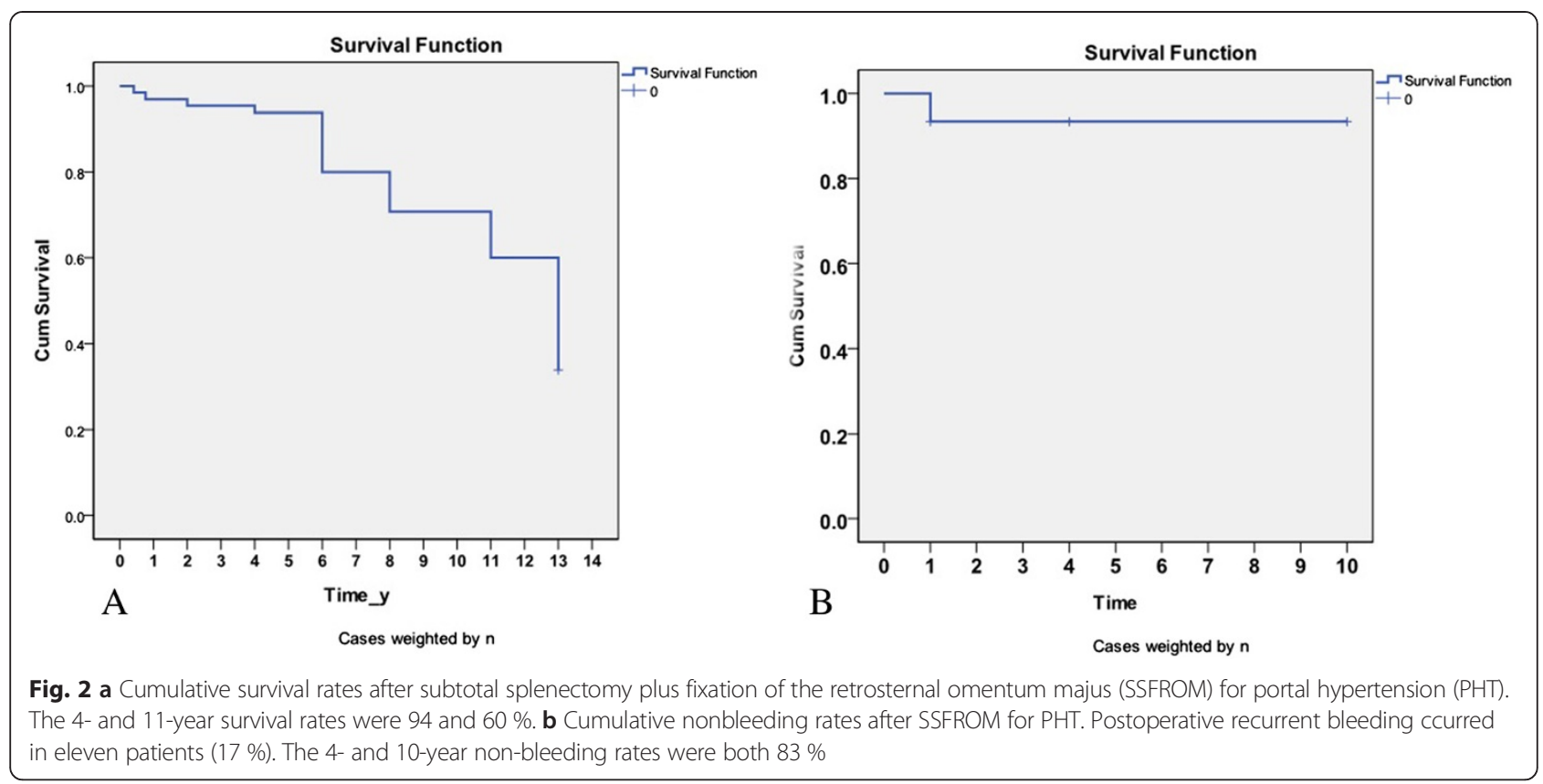

Fig. 2b). No patients have died from esophagogastric variceal bleeding to data. Post-operative endoscopy of the stomach showed exacerbation of esophagogastric varices in 11 patients and prophylactic endoscopic injection sclerotherapy was performed, after which neither re-bleeding nor re-exacerbation occurred. Portal thrombosis occurred in six patients $(9 \%)$, from preoperative partial portal thrombosis and post-operative exacerbation to complete occlusion of the portal vein in two patients (Fig. 3).

\section{Change in peripheral blood cells}

The white blood cell and platelet counts were associated with a significant rise 6 years post-operatively in

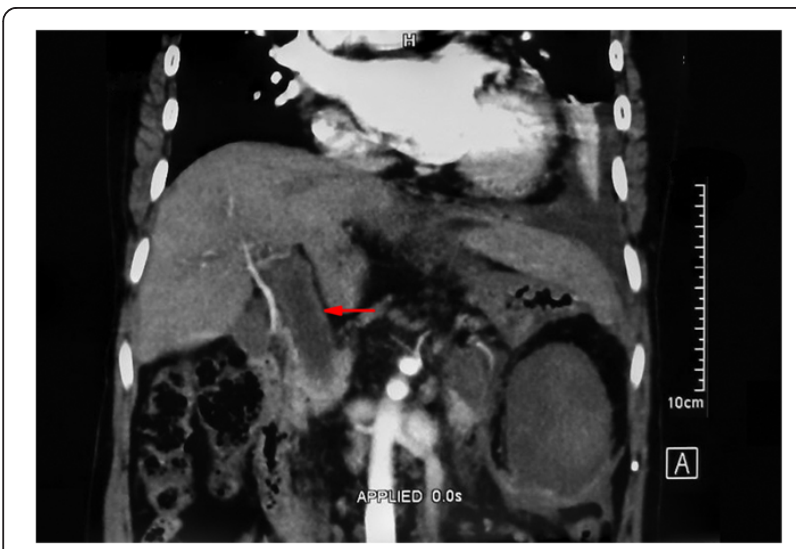

Fig. $3 \mathrm{CT}$ scan showed that patients was complicated with portal thrombosis at 4.5 months post-operatively
48 patients and 11 years in 16 patients post-operatively $(P<0.01$; Fig. 4a).

\section{Change in hemodynamics of the splenic artery and portal} vein

There were significant differences between the pre- and post-operative portal venous diameter, portal venous flow volume, and splenic artery flow volume $(P<0.01$; Fig. 4b, c).

\section{Change in splenic volume}

There were significant differences between the pre- and post-operative splenic length, splenic thickness, and splenic square area $(P<0.01$; Fig. $4 d$, e).

\section{Change in immunologic and related indices}

There were no significant differences between the IgA, IgM, IgG, and Tuftsin levels pre- and post-operatively ( $\mathrm{P}>0.05$; Fig. 4f, g). The serum levels of M-CSF and GMCSF were decreased significantly, and there was a significant difference between the pre- and post-operative values $(P<0.01$; Fig. $4 \mathrm{~h})$. The proportion of CD3+ T cells, CD4+ $\mathrm{T}$ cells, and CD8+ T cells was increased post-operatively compared with pre-operative values $(P<0.01$; Fig. $4 \mathrm{i})$.

\section{Change in splenic function}

A CT scan revealed that the blood supply of the residual spleen was normal (Fig. 5a, b). Isotope scanning with ${ }^{99} \mathrm{~m}$ Tc showed that the residual splenic phagocytic function had recovered (Fig. 5c). 

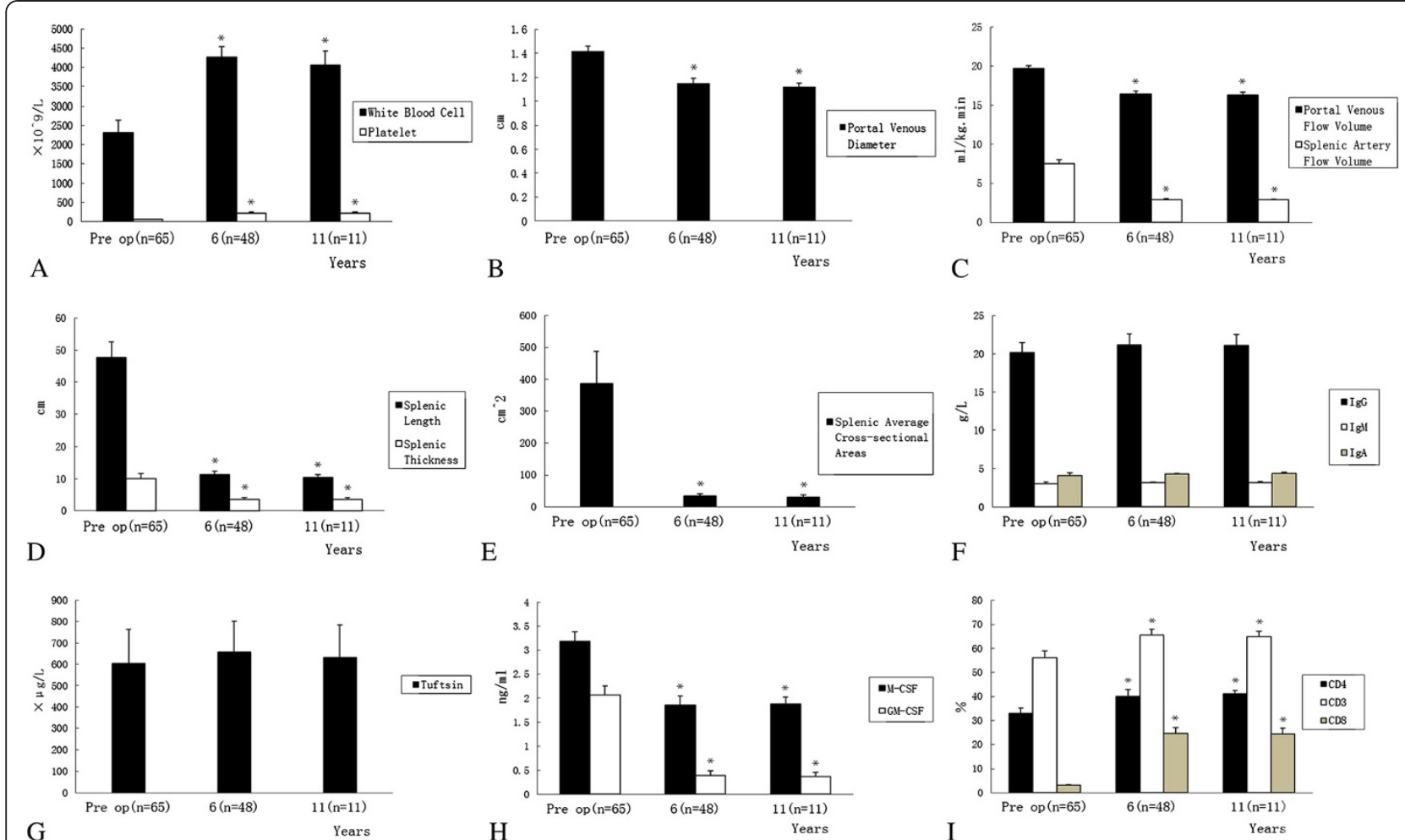

Fig. 4 a Changes in the white blood cell (WBC) count and platelet count at 6 - and 11 -years, after SSFROM for PHT $(* P<0.01)$. b Change in portal venous diameter at 6 - and 11 -years, after SSFROM for PHT ( $\left.{ }^{*} P<0.01\right)$. c Changes in portal venous flow volume, and splenic artery flow volume at 6- and 11-years, after SSFROM for PHT ( $\left.{ }^{*} P<0.01\right)$. $\mathbf{d}$ Changes in splenic length and splenic thickness at 6- and 11-years, after SSFROM for PHT

$\left({ }^{*} P<0.01\right)$. e Change in splenic square area. at 6 - and 11 - years, after SSFROM for PHT $\left({ }^{*} P<0.01\right)$. $\mathbf{f}$ Changes in levels of serum $\lg \mathrm{A}$, $\lg \mathrm{M}$ and $\lg \mathrm{g}$ at 6 and 11 years, after SSFROM for PHT ( $\left.{ }^{*} P>0.05\right)$. $\mathbf{g}$ Change in levels of serum Tuftsin at 6 and 11 years, after SSFROM for PHT $\left(^{*} P>0.05\right)$. $\mathbf{h}$ Changes in levels of serum macrophage colony-stimulating factor (M-CSF) and granulocyte-macrophage colony-stimulating factor and (GM-CSF) at 6 and 11 years, after SSFROM for PHT ( $\left.{ }^{*} P<0.01\right)$. i Changes in proportion of serum CD3+ T cells, CD4+ T cells, and CD8+ T cells at 6 and 11 years, after SSFROM for PHT $\left({ }^{*} P<0.01\right)$

\section{Portal-azygous collateral circulation}

Fifteen patients had indwelling catheters placed in the omental vein 1 month post-operatively. DSA showed that omental venous blood flowed into the superior vena cava. These results confirmed an established collateral circulation between the portal vein and superior vena cava (Fig. 5d).

\section{Discussion}

PHT is a clinical syndrome caused by the continual rise in portal vein pressure, and is closely associated with the incidence and mortality of chronic liver disease. The severity of PHT is indicated by a hepatic portal venous pressure gradient (difference between hepatic sinal portal wedge pressure and free hepatic venous pressure) [6]. Under high pressure, reactive changes are observed in the intra- and extra-hepatic vasculature, such as shear stress, vascular proliferation, and neovascularization. Collateral circulation plays an important role in buffering portal pressure [7]. Portal-systemic collateral vascular resistance and vasoconstrictor responsiveness are crucial in PHT and control of variceal bleeding [8]. PHT induces the formation of portal systemic collaterals. Revealing the developmental change in portal-systemic collaterals is important for future therapy [9]. The incidence of gastric varices is $25 \%$ and the rate of gastric variceal hemorrhage is less than esophageal variceal hemorrhage [10]. As PHT develops, the formation of collateral vessels and arterial vasodilation progresses, which results in increased blood flow to the portal circulation [11]. The special anatomic structure of the esophageal venous plexus results in easy, and even fatal, variceal bleeding. According to statistics, esophageal varices are detected in approximately $50 \%$ of cirrhosis patients, and $5-15 \%$ of cirrhotic patients have newly formed varices or worsening of varices each year [12]. Thus, an understanding of the pathogenesis of the collateral circulation and compensatory mechanism in patients with PHT is crucial.

The spleen is the only solid organ of the portal venous system. In patients with PHT, portal venous blood returns flow and the splenic functions as an auxiliary capacitance system which compensates for the increase 


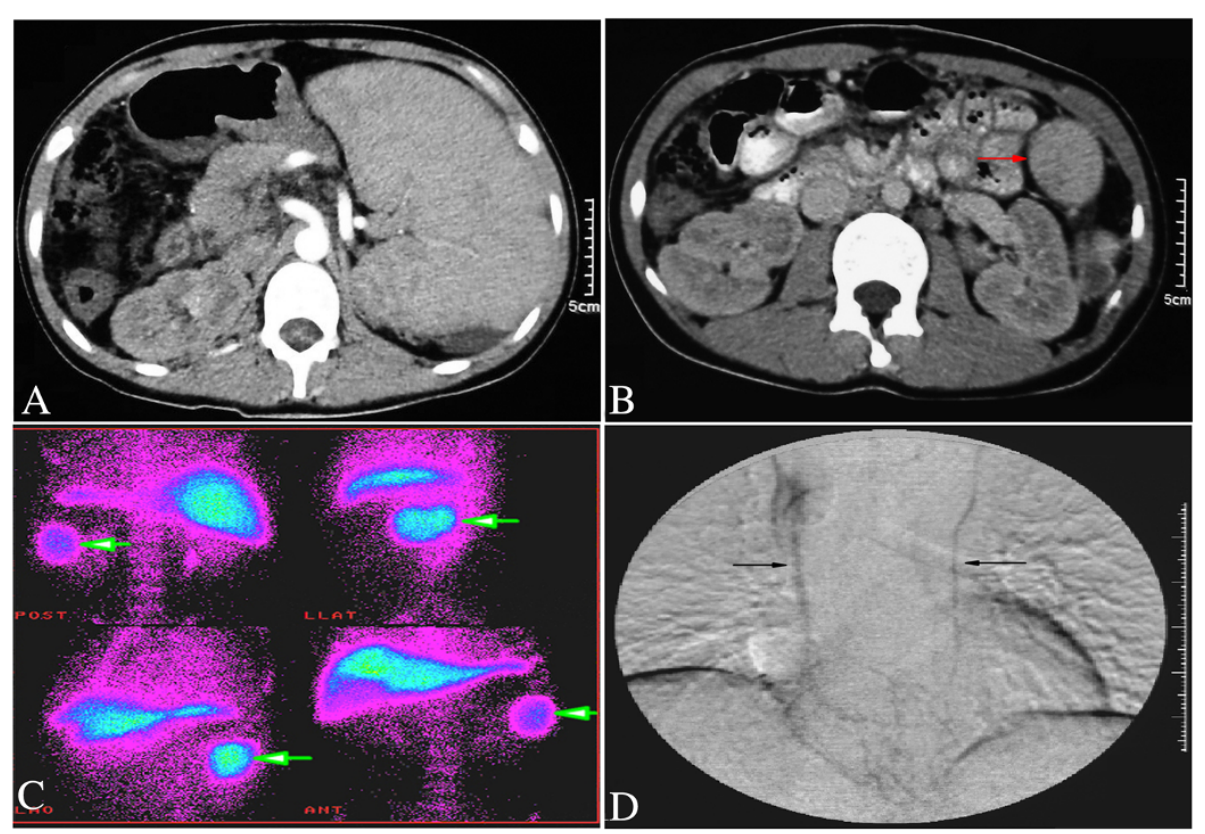

Fig. $\mathbf{5}$ a $C T$ scan revealed splenomegaly pre-operatively. $\mathbf{b} \subset T$ scan revealed that the blood supply of the residual spleen was normal (arrow) at 1-year, after SSFROM for PHT. c Isotope scanning with 99mTc showed that the residual splenic phagocytic function had recovered (arrow) at 4-years, after SSFROM for PHT. d DSA showed an established collateral circulation between the portal vein and superior vena cava (arrow) at 1 month, after SSFROM for PHT

in portal pressure that may cause splenomegaly [13]. The splenic angiogenesis can regulate the portal systemic collateral circulation and increase splenic blood flow, which is a pathophysiologic hallmark of the spleen $[14,15]$. Traditionally, splenomegaly has been thought to be due to passive splenic congestion. Recent research suggests that factors of splenomegaly in portal hypertension include not only spleen congestion, as traditionally thought, but also enlargement and hyperactivation of the splenic lymphoid tissue, as well as increased angiogenesis and fibrogenesis. Splenomegaly will not occur if the spontaneous portal systemic shunt exists. Therefore, portal systemic collaterals and splenomegaly are the compensation of PHT. Of portal hypertensive patients with splenomegaly and hypersplenism, $64 \%$ have a simultaneous decrease in various blood cells, $36 \%$ have a decrease in single type blood cells, and $52 \%$ of patients have bone marrow hyperplasia. Therefore, a decrease in peripheral blood cells and bone marrow hyperplasia are crucial indices of hypersplenism $[16,17]$. In splenomegaly, the medial ramus of perisplenic ligaments (lienorenal, splenophrenic, and splenocolic ligaments) have abundant vessels, and extensive collateral circulation is established between the retroperitoneal space and pericardium, thus the spleen can be called a "shunt bridge". The major innovation of the Warren operation utilizes the spleen as a shunt bridge, which shunts blood flow to the stomach and spleen to decrease pressure selectively [18].
In general, vein ligation under endoscopy and injection sclerotherapy are the preferred hematostatic method for esophagogastric variceal bleeding, which have the advantages of minimal trauma and rapid control of hemorrhage compared with surgery; however, for some patients, the long-term recurrent bleeding rate is higher than surgery $[19,20]$. For surgeons, the portosystemic shunt should be performed after a transjugular intrahepatic portosystemic shunt and before a liver transplantation [21]. In a study by Orloff et al., [22] a portal-systemic shunt was used to treat 200 patients with portal vein thrombosis (extrahepatic PHT). Actuarial 5-, 10-, and 15-year post-operative survival rates were $99 \%, 97 \%$, and $95 \%$, respectively. No patient developed portal-systemic encephalopathy. Gajin et al., [23] compared different surgeries in PHT patients and found that in the group of patients treated by distal splenorenal shunt with partial spleen resection, only one patient had splenomegaly post-operatively ( $5 \%$ ), while in the group of patients treated by a distal splenorenal shunt only, there were 13 patients with splenomegaly $(68 \%)$. There was a significant statistical difference in platelet count and splenic volume, which revealed that distal splenorenal shunt (Warren) plus partial spleen resection was superior to the original Warren. Sretenovic et al., [24] performed distal splenorenal shunts plus partial resection of the spleen in 16 children with extrahepatic PHT. The post-operative 2-year recurrent bleeding rate was $5 \%$ and the incidence of thrombosis was $7 \%$. This surgical 
technique was applicable to patients with extrahepatic PHT. Because of favorable liver function and without cirrhosis, the post-operative long-term survival rate was higher and the recurrent bleeding rate was lower. However, for patients with post-hepatitis cirrhosis, liver cancer and liver failure may occur due to poor liver function and the long-term survival rate decreased. However, for cirrhosis after hepatitis B infection, because of unfavorable liver function, there was an increased probability for hepatomas and liver failure, and the post-operative long-term survival rate decreased. Therefore, the survival and recurrent bleeding rates depended on liver function.

SSFROM include the following. 1. Subtotal splenectomy: The short gastric vessels and splenic artery stem were ligated, preserving the lower splenic pole, and the residual spleen was supplied by the left splenic omentum and splenocolic vessels. The residual spleen and partial omentum were fixed into the retroperitoneum. On the one hand, the residual spleen can eliminate the highpressure state, and prevent recurrence of splenomegaly and hypersplenism. On the other hand, as a bridge of the portal-systemic shunt, the residual spleen can shunt the high-pressure venous flow into the systemic circulation. 2. Pericardial devascularization: Hemostasis of vascular devascularization, especially the high esophageal branch, which was crucial before the portal-systemic shunt was established. 3. Fixation of the retrosternal omentum majus: Collateral circulation was established between the omentum majus and azygos vein; this decreases the bleeding risk of a natural portal-azygous collateral pathway. Shunt flow volume can follow up the portal pressure gradient and thoracic coeliac pressure difference in self-regulation, without concern about stenosis of the anastomotic stoma and embolization occurring. Post-operative DSA showed that the portal-azygous collateral circulation was established. Color Doppler ultrasound suggested that portal blood flow volume and portal venous diameter have a significant constriction or decrease compared with pre-operatively. The recurrent bleeding rate was $17 \%$ without hepatic encephalopathy. It was revealed that this surgical technique has the longterm effect of shunting, decreased pressure, and controlled bleeding. The long-term follow-up of patients after subtotal splenectomy showed that residual splenic blood flow decreased; no residual splenic volume enlargement occurred. Radioisotope scanning showed that residual splenic phagocytosis was normal. Residual splenic immune indices were the same as pre-operatively. Post-operative peripheral white blood cell and platelet counts were associated with a significant pre-operative rise. Not only was the residual splenic blood flow reduced, but hypersplenism was corrected and residual splenic phagocytosis and immune function still existed. The level of serum T lymphocyte subpopulation was higher and the level of serum M-CSF and
GM-CSF was lower post-operatively compared with preoperatively. The results suggested that residual splenic tissue of relieving high pressure can enhance the immune reaction and weaken the inflammatory reaction. Our previous study showed that there was no significant difference in the content of collagen, elasticity, and reticular fiber between the residual spleen and splenomegaly. Electron microscopy showed that the ultrastructure of lymphocytes was improved. Immunohistochemical staining showed that counts per unit area of $\mathrm{T}$ and $\mathrm{B}$ lymphocytes and macrophages increased markedly post-operatively compared with pre-operatively $[25,26]$. Thus, it can be seen that the anatomic and physiologic foundation of subtotal splenectomy provides valuable clinical information for splenic preservation. Our follow-up results (recurrent bleeding rate, and hemodynamic, hematologic, and immunologic indices) were nearly in agreement with that reported by Hase et al [27]. There is a difference in survival rate, which may be caused by different liver function. Hemorrhoidal bleeding was not observed in our study. Our data showed that the major reasons for massive post-operative bleeding were as follows: 1 . In the short-term, collateral circulation was not established between the portal and azygos veins, and the portal venous pressure of the patients after disconnection showed a transient increase. and 2. After disconnection, blood flow to the lower esophagus and gastric wall had a high dynamic state, which induced a submucosal arteriovenous shunt, mucosal ischemia, hypofunction of the barrier, and formed the pathogenic basis of mucosal lesions. Our data also showed that the incidence of post-operative portal thrombosis was $9 \%$, which was lower than other reports [28-32]. The analyses were related to protection of the partial spleen and administration of a corresponding anticoagulant measure(low-molecular weight heparin calcium [5000 U subcutaneously] starting on the 2nd day after surgery (daily for 2 weeks). Then, warfarin or rivaroxaban was taken orally once a day for 3 months). In the current study, the patients with postoperative portal thromboses were treated with anticoagulation and antiagglutinating as well as thrombolysis, and was absorbed completely or the improvement of absorption within 2-4 weeks. In our opinion, compared with the Warren operation, SSFROM is a simple operation, is easy to popularize, and can obtain the same clinical effect; therefore it is worth popularizing. However, it must be emphasized that the patients in this study were select patients, class A or B according to the Child-Pugh classification, and controlled content of the virus can improve the long-term survival rate.

\section{Conclusion}

SSFROM provides long-term hemostasis for esophagogastric variceal bleeding in PHT and corrects hypersplenism. 


\section{SSFROM is an effective treatment for patients with PHT in whom long-term survival is expected.}

\section{Abbreviations}

PHT: Portal hypertension; SSFROM: Subtotal splenectomy plus fixation of the retrosternal omentum majus; M-CSF: Macrophage colony-stimulating factor; GM-CSF: Granulocyte-macrophage colony-stimulating factor; DSA: Digital subtraction angiography.

\section{Competing interests}

The authors declared no potential conflicts of interest with respect to the research, authorship, and/or publication of this article.

\section{Authors' contributions}

Drs WZ, TW participated in the clinical specimen and data collection. Drs LW YX, FJ performed data analysis and drafted the manuscript. Drs HC, WH and JZ conceived the study, writing and final approval of the version to be published. All authors read and approved the final manuscript.

\section{Acknowledgments}

We would like to thank Xiangyun Li, Prof. MD for technical assistance, Department of Health Statistics, Weifang Medical University.

\section{Funding}

The authors received no financial support for the research, authorship, and/ or publication of this article.

\section{Author details}

'Center of General Surgery, The 89th Hospital of People's Liberation Army, West Beigong Road 256, Weifang 261021, China. ${ }^{2}$ Department of Postgraduate, Weifang Medical University, Weifang 261042, China. ${ }^{3}$ Department of General Surgery, Changyi People's Hospital, Changyi 261300, China. ${ }^{4}$ Department of General Surgery, Weifang Traditional Chinese Medicine Hospital, Weifang 261041, China. ${ }^{5}$ Department of Pathology, The 89th Hospital of People's Liberation Army, Weifang 261021, China.

\section{Received: 16 February 2015 Accepted: 20 July 2015}

Published online: 24 July 2015

\section{References}

1. Wu J, Li Z, Wang Z, Han X, Ji F, Zhang WW. Surgical and endovascular treatment of severe complications secondary to noncirrhotic portal hypertension: experience of 56 cases. Ann Vasc Surg. 2013;27(4):441-6.

2. Batista-Neto J, Tognetti LB, Ribeiro LT, Balwani Mdo C, Muritiba T, Alves EE. Evolutional profile of the esophageal varices after splenectomy associated with ligation of the left gastric vein and sclerotherapy in schistosomal portal hypertension. Arq Bras Cir Dig. 2013;26(1):49-53.

3. Kim SJ, Oh SH, Jo JM, Kim KM. Experiences with endoscopic interventions for variceal bleeding children with portal hypertension: a single center study. Pediatr Gastroenterol Hepatol Nutr. 2013;16(4):248-53.

4. Wolff M, Hirner A. Surgical treatment of portal hypertension. Zentralbl Chir. 2005;130(3):238-45.

5. Huang FR, Chu HB, Pan RW, Sun XJ, Li PC, Li YL. Treatment for patients with portal hypertension who underwent subtotal splenectomy and fixation of substernal omentum majus. Chin J Hepatobiliary Surg. 2002;8(9):564-5.

6. Burroughs AK, Thalheimer U. Hepatic venous pressure gradient in 2010: optimal measurement is key. Hepatology. 2010:51(6):1894-6

7. Kim MY, Baik SK. Pathophysiology of portal hypertension, what's new? Korean J Gastroenterol. 2010;56(3):129-34.

8. Huang HC, Wang SS, Lee JY, Chen YC, Lee FY, Lin HC, et al. Simvastatin effects on portal-systemic collaterals of portal hypertensive rats. J Gastroenterol Hepatol. 2010;25(8):1401-9.

9. Chan CC, Tsai SC, Cheng LY, Lee FY, Lin HC. Hemodynamic assessment of the development of portal-systemic collaterals in portal hypertensive rats. Dig Dis Sci. 2011;56(2):417-24.

10. Kim JN, Sohn KM, Kim MY, Suk KT, Jeong SW, Jung HE, et al. Relationship between the hepatic venous pressure gradient and first variceal hemorrhage in patients with cirrhosis: a multicenter retrospective study in Korea. Clin Mole Hepatol. 2012;18(4):391-6.

11. Iwakiri Y. Pathophysiology of portal hypertension. Clin Liver Dis. 2014;18:281-91.
12. Maruyama H, Yokosuka O. Pathophysiology of portal hypertension and esophageal varices. Int J Hepatol. 2012;2012:895787.

13. Silva AR, Kriguer-Júnior RJ, Serigiolle LC, Gomes HM, Rodrigues DA, Leme PL. Increase in spleen volume of rats on experimental model of pre-hepatic portal hypertension. Arq Bras Cir Dig. 2013;26(3):206-12.

14. Coulon S, Heindryckx F, Geerts A, Van Steenkiste C, Colle I, Van Vlierberghe $\mathrm{H}$. Angiogenesis in chronic liver disease and its complications. Liver Int. 2011;31(2):146-62.

15. Tiani C, Garcia-Pras E, Mejias M, de Gottardi A, Berzigotti A, Bosch J, et al. Apelin signaling modulates splanchnic angiogencesis and portosystemi collateral vessel formation in rats with portal hypertension. J Hepatol. 2009;50(2):296-305.

16. Lu YF, Li XQ, Han XY, Gong XG, Chang SW. Peripheral blood cell variations in cirrhotic portal hypertension patients with hypersplenism. Asian Pac J Trop Med. 2013;6(8):663-6.

17. Jeker R. Hypersplenism. Ther Umsch. 2013;70(3):152-6.

18. Warren WD, Zeppa R, Fomon JJ. Selective trans-splenic decompression of gastroesophageal varices by distal splenorenal shunt. Ann Surg. 1967;166(3):437-55.

19. Ibrahim M, El-Mikkawy A, Mostafa I, Devière J. Endoscopic treatment of acute variceal hemorrhage by using hemostatic powder TC-325: a prospective pilot study. Gastrointest Endosc. 2013;78(5):769-73.

20. Elwakil R, Reda MA, Abdelhakam SM, Ghoraba DM, Ibrahim WA. Causes and outcome of upper gastrointestinal bleeding in Emergency Endoscopy Unit of Ain Shams University Hospital. J Egypt Soc Parasitol. 2011;41(2):455-67.

21. Puhl G, Gül S, Neuhaus P. Portosystemic shunt surgery between TIPS and liver transplantation. Chirurg. 2011;82(10):898-905.

22. Orloff MJ, Orloff MS, Girard B, Orloff SL. Bleeding esophagogastric varices from extrahepatic portal hypertension: 40 years' experience with portal-systemic shunt. J Am Coll Surg. 2002;194(6):717-28.

23. Gajin P, Radević B, Nenezić D, llijevski N, Jesić-Vukićević R, Radak D. Distal splenorenal shunt with partial spleen resection. Srp Arh Celok Lek. 2007:135(5-6):293-7.

24. Sretenovic AL, Perišić V, Krstić Z, Vujović D, Pavićević P, Stanisavljević $D$, et al. Warren shunt combined with partial splenectomy for children with extrahepatic portal hypertension, massive splenomegaly, and severe hypersplenism. Surg Today. 2013;43(5):521-5.

25. Chu H, Liu X, Zhao J, Xu Y, Wang L, Wang T, et al. Subtotal splenectomy for splenomegaly in cirrhotic patients. Int J Clin Exp Pathol. 2014;7(8):4981-90

26. Chu HB, Zhang TG, Zhao JH, Jian FG, Xu YB, Wang T, et al. Assessment of immune cells and function of the residual spleen after subtotal splenectomy due to splenomegaly in cirrhotic patients. BMC Immunol. 2014;15(1):42.

27. Hase R, Hirano S, Kondo S, Okushiba S, Morikawa T, Katoh H. Long-term efficacy of distal splenorenal shunt with splenopancreatic and gastric disconnection for esophagogastric varices in patients with idiopathic portal hypertension. World J Surg. 2005;29(8):1034-6.

28. Harding DJ, Perera MT, Chen F, Olliff S, Tripathi D. Portal vein thrombosis in cirrhosis: Controversies and latest developments. World J Gastroenterol. 2015;21(22):6769-84

29. Lai W, Lu SC, Li GY, Li CY, Wu JS, Guo QL, et al. Anticoagulation therapy prevents portal-splenic vein thrombosis after splenectomy with gastroesophageal devascularization. World I Gastroenterol. 2012;18(26):3443-50

30. Nery F, Chevret S, Condat B, de Raucourt E, Boudaoud L, Rautou PE, et al. Causes and consequences of portal vein thrombosis in 1243 patients with cirrhosis: results of a longitudinal study. Hepatology. 2015;61(2):660-7.

31. Abdel-Razik A, Mousa N, Elhelaly R, Tawfik A. De-novo portal vein thrombosis in liver cirrhosis: risk factors and correlation with the Model for End-stage Liver Disease scoring system. Eur J Gastroenterol Hepatol. 2015;27(5):585-92

32. Sun L, Zhou H, Gu L, Jiang C, Liu Y, Xu Q. Effects of surgical procedures on the occurrence and development of postoperative portal vein thrombosis in patients with cirrhosis complicated by portal hypertension. Int J Surg. 2015;16(Pt A):31-5 